\title{
Evaluation of Prognostic Factors Associated With Differentiated Thyroid Carcinoma With Pulmonary Metastasis
}

\author{
Mohsen Qutbi, MD, * Babak Shafeie, MD, * Mahasti Amoui, MD, † Faraj Tabeie, PhD, * \\ Zahra Azizmohammadi, MD, * Ali Mahmoud-Pashazadeh, MSc, $\neq$ Hamid Javadi, MD, $\S$ \\ Majid Assadi, MD, $\neq$ and Isa Neshandar Asli, MD*
}

\begin{abstract}
Background: Because one of the major sites for metastasis of thyroid cancers is the lung, studying the pattern of pulmonary metastasis may provide useful information for the effective treatment of these patients. In this study, by assessing the metastasis pattern, we aimed to identify the factors that may affect prognosis and response to treatment in patients with differentiated thyroid carcinoma (DTC) with pulmonary metastasis.

Methods: This retrospective study included 75 patients with DTC with pulmonary metastasis who were referred to our nuclear medicine section over a period of 10 years. The data obtained were analyzed with regard to response to treatment to assess the effects of the included factors on prognosis.

Results: Of the 1746 patients referred to our section, 75 (4.3\%) had pulmonary metastasis. According to the pattern of pulmonary metastasis, they were divided into 4 groups: nodular, diffuse, combined, and other. The mean age of the patients was $43.8 \pm 18.5$ years. After the follow-up, 58 patients survived, 14 of whom responded to the treatment. The mean number of radioiodine therapy sessions that the patients received was $3.2 \pm 2$, and the mean cumulative dose was $554.7 \pm 387.8 \mathrm{mCi}$. Statistical analysis of the data revealed that there was no significant difference in the response to treatment between patients with different patterns of pulmonary metastasis $(P>0.3)$. However, significant differences were reported in the response to treatment between patients with papillary thyroid carcinoma and those with follicular thyroid carcinoma $(P<0.03)$. The 1-, 5-, and 9-year survival rates were reported as $98 \%, 76 \%$, and $51 \%$, respectively.
\end{abstract}

Conclusions: Patients with DTC with pulmonary metastasis have a relatively favorable prognosis and response rate, as well as longer survival. The type of DTC is the only factor that affects the response to treatment.

Key Words: differentiated thyroid cancer, prognosis, pulmonary metastasis, response to treatment

(Clin Nucl Med 2016;41: 917-921)

$\mathrm{D}$ ifferentiated thyroid carcinoma (DTC) is of the most curable endocrine cancers; it can be classified as papillary thyroid carcinoma (PTC) and follicular thyroid carcinoma (FTC). This is a slowly progressing disease that has been reported to have a 10 -year survival rate of more than $80 \%$. ${ }^{1}$ The reported incidence of DTC has been increasing worldwide; this is partly attributable to the

Received for publication March 10, 2016; revision accepted September 8, 2016. From the *Department of Nuclear Medicine, Taleghani Hospital, Shahid Beheshti University of Medical Sciences, Tehran; $†$ Department of Nuclear Medicine, Shohada-e-Tajrish Medical Center, Shahid Beheshti University of Medical Sciences, Tehran; $¥$ The Persian Gulf Nuclear Medicine Research Center, Bushehr University of Medical Sciences, Bushehr; and §Golestan Research Center of Gastroenterology and Hepatology (GRCGH), Golestan University of Medical Sciences, Gorgan, Iran.

This study was the postgraduate thesis of M.Q. and was supported by the Shahid Beheshti University of Medical Sciences (grant 2098).

Conflicts of interest and sources of funding: none declared.

Correspondence to: Majid Assadi, MD, The Persian Gulf Nuclear Medicine Research Center, Bushehr University of Medical Sciences, Moallem Street, Bushehr 7513934698, Iran. E-mail: assadipoya@yahoo.com.

Copyright (C) 2016 Wolters Kluwer Health, Inc. All rights reserved.

ISSN: 0363-9762/16/4112-0917

DOI: $10.1097 /$ RLU.0000000000001426 development of diagnostic techniques, ${ }^{2-4}$ which have made highsensitivity diagnosis feasible and partly attributable to exposure to radiation in our environment as well as risky living habits. ${ }^{5,6}$ While DTC is mainly limited to the thyroid gland, it has been reported that distant metastasis, particularly pulmonary metastasis, occurs in $4 \%$ to $23 \%$ of DTC patients, ${ }^{7-10}$ which may reduce the associated survival rate and prognosis. In some rare cases of DTC, the brain, breast, liver, kidney, muscle, and skin are also involved. The presence of distant metastases is of pivotal importance for physicians, because it can be an indicator of poor survival, as only half of the patients who have metastasis may survive for more than 10 years. ${ }^{11}$

Several studies have been performed to assess the outcome and prognosis of DTC patients with pulmonary metastasis: age, thyroglobulin ( $\mathrm{Tg}$ ) levels cumulative dose of radioiodine, and size of the tumor were found to be important prognostic factors.

As recognizing the patterns of pulmonary metastasis in DTC patients has a major impact on the prognosis of patients and can help physicians with their clinical decision making, the objective of this study was to assess the pattern of metastasis and identify the prognostic factors associated with survival and response to treatment in DTC patients with pulmonary metastases.

\section{MATERIALS AND METHODS}

The current survey is a retrospective cross-sectional study that assesses the prognostic value of the pattern of pulmonary metastasis in DTC patients and its impact on outcome and response to treatment with radioiodine.

The data for this study were extracted from the medical records of DTC patients with pulmonary metastases who were hospitalized at the Department of Nuclear Medicine of a tertiary university-affiliated hospital between 2005 and 2015. We included only DTC patients who underwent total thyroidectomy and received a diagnosis of pulmonary metastasis based on posttreatment wholebody scan or chest CT scan, chest radiography, or radioactive iodine whole-body scan during follow-up. The disease status was monitored by whole-body radioiodine scanning and measuring the serum $\mathrm{Tg}$ level.

This study complies with the Declaration of Helsinki, and it was approved by the institutional ethics committee of our university.

\section{Statistical Analysis}

All data are presented as the mean $\pm \mathrm{SD}$, with ranges given when appropriate. Continuous variables were evaluated using the unpaired $t$ test, and categorical variables were evaluated using the $\chi^{2}$ analysis. A survival analysis using Kaplan-Meier test was also carried out. To compare survival between groups, the log-rank test was applied.

$P<0.05$ was considered to be statistically significant. The SPSS for Windows software package (Release 22; SPSS Inc, Chicago, Ill) was applied for the statistical analysis.

\section{RESULTS}

Of the 1746 patients who were referred to our nuclear medicine section and hospitalized for DTC, 75 (4.3\%) had pulmonary 
metastases and were included in this study. Of the 75 patients, 60 $(80 \%)$ were female, and $15(20 \%)$ were male (age, $13-79$ years; mean age, $43.8 \pm 18.5$ years). Based on the pathological characteristics of the tumor, 61 patients $(81.3 \%$ ) received a diagnosis of PTC (46 females and 15 males), and 14 patients (18.7\%) received a diagnosis of FTC (all females). The average number of hospitalizations during which radioiodine treatment was administered was $3.2 \pm 2$ (median, 3): it was $3.2 \pm 1.9$ for female patients and $3.1 \pm 2.3$ for male patients. Moreover, the average number of hospitalizations (with radioiodine treatment) was $3.2 \pm 2.1$ in patients with PTC and $3.0 \pm 1.2$ in patients with FTC. The average cumulative dose was $554.7 \pm 387.8 \mathrm{mCi}$ (range, $100-1650 \mathrm{mCi}$ ). The average cumulative dose was $551 \mathrm{mCi}$ in female patients and $566 \mathrm{mCi}$ in male patients. Moreover, it was $560 \mathrm{mCi}$ in patients with PTC and $528 \mathrm{mCi}$ in patients with FTC. Of the total patients, 67 patients $(89.3 \%)$ were positive, and 8 patients $(10.7 \%$ ) were negative for cervical lymph node involvement. Among the female patients, 55 patients $(91.7 \%)$ were positive, and 5 patients $(8.3 \%)$ were negative for cervical lymph node involvement. Furthermore, among the male patients, 12 $(80 \%)$ were positive, and $3(20 \%)$ were negative for cervical lymph node involvement. Among patients with PTC, 55 (90.2\%) were positive, and $6(9.2 \%)$ were negative for lymph node involvement, and in patients with FTC, 12 patients $(85.7 \%)$ were positive, and $2(14.3 \%)$ were negative for lymph node involvement. With regard to distant metastasis other than pulmonary metastasis, 16 patients $(21.3 \%)$ received a diagnosis of bone metastases, and 2 patients $(2.7 \%)$ received a diagnosis of liver metastasis. Among the female patients, $10(16.7 \%)$ had bone metastasis, and $2(3.3 \%)$ had liver metastasis. Among the male patients, $6(40 \%)$ had bone metastasis with no liver metastasis. Among the patients with PTC, $11(18 \%)$ had bone metastasis, and $1(1.6 \%)$ had liver metastasis. Among the patients with FTC, $5(35.7 \%)$ had bone metastasis, and 1 (7.1\%) had liver metastasis. With regard to the pattern of pulmonary metastasis, 42 patients $(56 \%)$ had a diffuse pattern, 19 patients $(25.3 \%)$ had nodular involvement, and $11(14.7 \%)$ had a combination of both; the remaining 3 patients $(4 \%)$ had other pattern. The diffuse pattern, nodal involvement, and combination of both were observed in 34 $(56.7 \%), 15(25 \%)$, and $8(13.3 \%)$ female patients, respectively, and $8(53.3 \%), 4(26.7 \%)$, and $3(20 \%)$ male patients, respectively; and $34(55.7 \%), 16(26.2 \%)$, and $8(13.1 \%)$ patients with PTC, respectively, and $8(57.1 \%), 3(21.4 \%)$, and $3(21.4 \%)$ patients with FTC, respectively.

Of the total of 75 patients who were included because they had pulmonary metastasis, 61 patients $(81.3 \%)$ had pulmonary metastasis at presentation; 14 patients $(18.7 \%)$ had no sign of pulmonary involvement, but at later stages of the disease, they received a diagnosis of pulmonary metastasis. The interval between the diagnosis of DTC and diagnosis of pulmonary metastasis was in the range of 6 to 48 months (mean, $12.6 \pm 20.4$ months; median, 17 months).

Of the 75 patients, 58 were followed up, 14 of whom responded to treatment, and 44 of whom did not respond to treatment. With regard to the patients who responded to the treatment, the average time from diagnosis of pulmonary metastasis to response to treatment was 8 to 48 months (mean, $20.8 \pm 13.6$ months). In this duration, the patients underwent 1 to 7 sessions of iodine therapy (mean, $2.5 \pm 1.6$; median, 2). Moreover, in this duration, the cumulative dose given to the patients ranged from 150 to $950 \mathrm{mCi}$ (mean, $387.5 \pm 214.4 \mathrm{mCi}$ ). With regard to the pattern of pulmonary involvement in these patients, 6 had a diffuse pattern, 4 had nodular involvement, and 4 had a combination of both.

Of the 58 patients who were followed up, 51 patients were alive, and 7 patients had died at the end of the follow-up period. Four patients died because of respiratory failure resulting from extensive pulmonary metastasis, 1 patient died because of extensive myocardial infarction, one because of cerebral metastasis, and also 1 patient died because of multiple bone metastases. Furthermore, of 4 patients who died as a result of respiratory failure, 2 patients

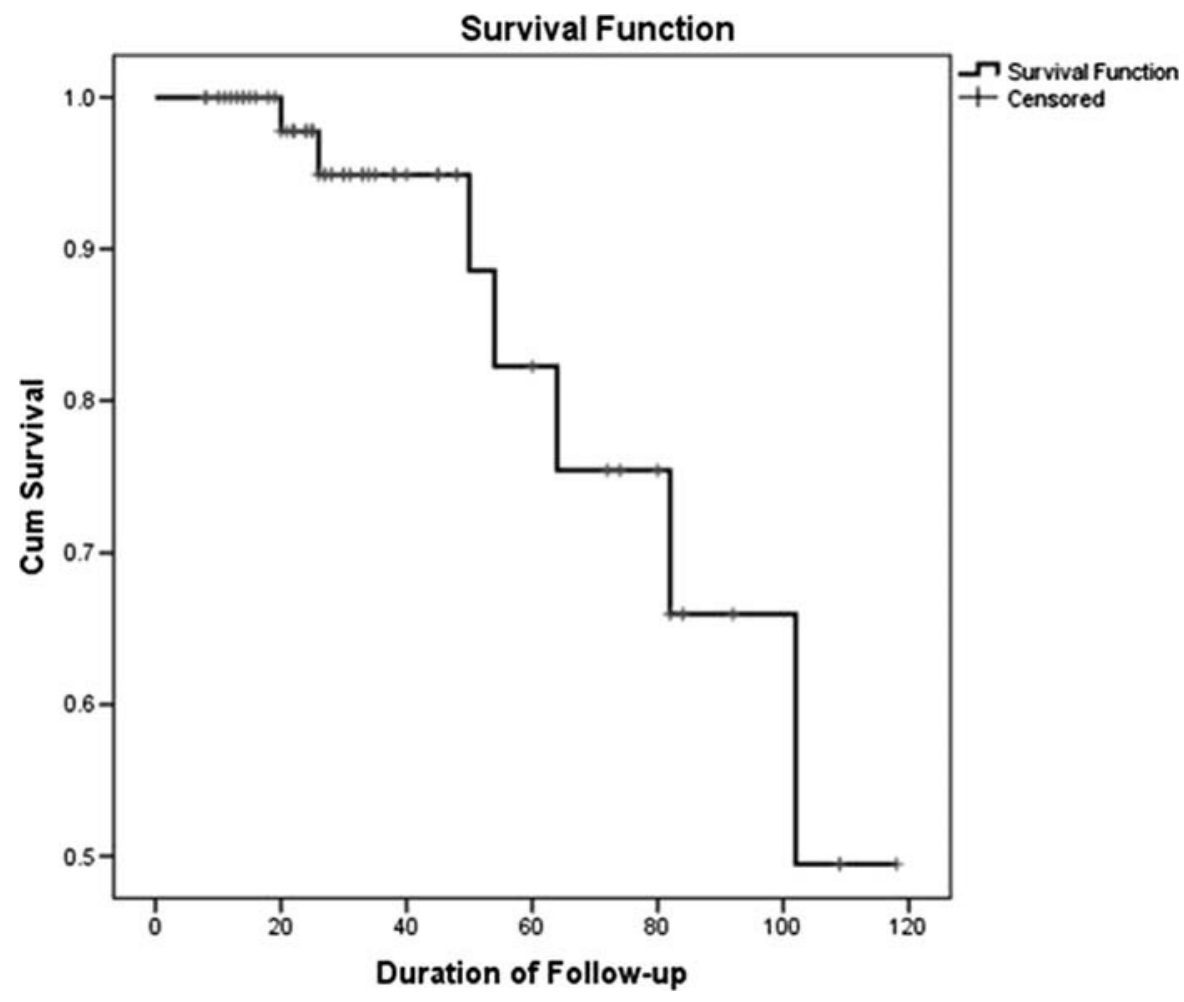

FIGURE 1. Survival rate of patients with DTC who present with pulmonary metastasis. 


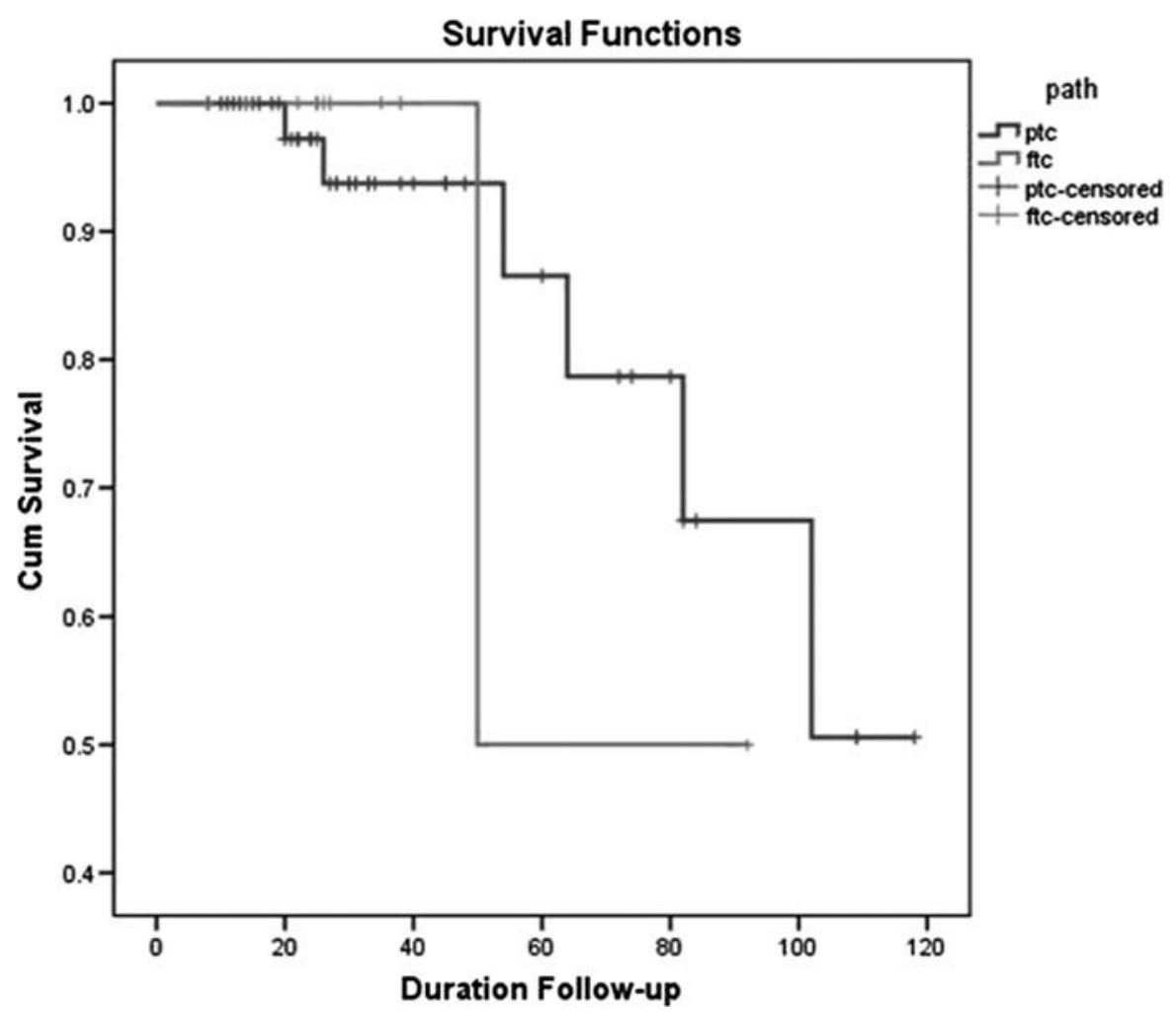

FIGURE 2. Survival rate of patients between 2 types of papillary and follicular thyroid cancer who present with pulmonary metastasis.

had bone metastases, and 1 had liver metastasis. There were no significant differences in cause of death and other clinical and paraclinical variables including age, sex, number of hospitalizations, average time from diagnosis of pulmonary metastasis to response to treatment, cumulative dose, the pattern of pulmonary involvement, being distant metastasis, and type of pathology of thyroid cancer $(P>0.05)$.
Statistical analysis of the data revealed that there was no significant difference in the response to treatment and also survival rate between patients with different patterns of pulmonary involvement $(P>0.3)$. A significant difference was found in the response to treatment between patients with PTC and patients with FTC $(P<0.03)$, but the survival rate between them was not significant statistically

\section{Survival Functions}

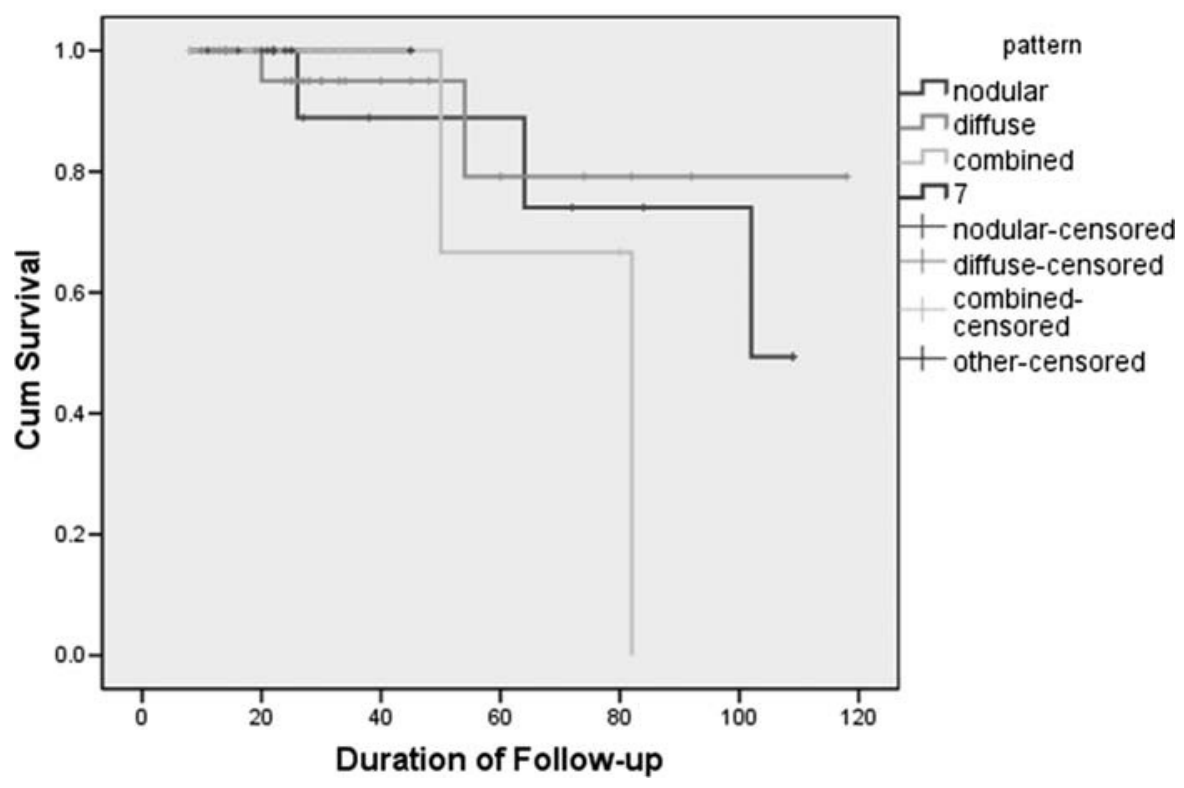

FIGURE 3. Survival rate of patients with DTC according to the pulmonary metastasis pattern. 


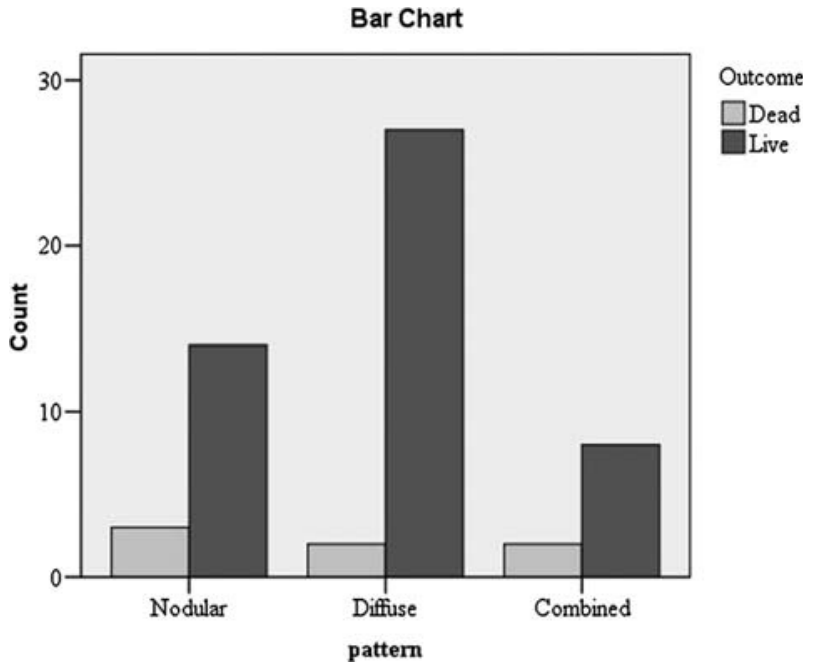

FIGURE 4. Outcome of radioiodine therapy in DTC patients according to the metastasis pattern.

$(P>0.05)$. The 1-, 5-, and 9-year survival rates were $98 \%, 76 \%$, and $51 \%$, respectively (Figs. $1-3$ ).

The outcomes of radioiodine therapy and response to treatment, according to the type of pulmonary involvement, are shown in Figures 4 and 5.

\section{DISCUSSION}

Given the importance of factors that affect the prognosis and survival of DTC patients, more knowledge about these factors can prove to be vital for better treatment planning, as well as improving adherence to treatment.

In DTC patients, early detection and treatment have been found to have a substantial effect on the survival rate of patients. It has been reported that a significant proportion of these patients have regional metastasis $(20 \%-50 \%)$ and distant metastasis $(3.3 \%$ $6 \%) .{ }^{12, P 3}$ Metastasis not only reduces survival but also can adversely affect the quality of life of these patients, who are mostly young.

The results of our study were in a good agreement with previous ones ${ }^{14}$; according to the previous findings, a considerable proportion of patients have metastasis at the time of diagnosis of thyroid cancers $(81 \%$ vs $75 \%)$. The number of patients who had metastasis at the time of diagnosis was higher in the FTC than in the PTC group. This finding emphasizes the importance of measuring the Tg level and radioiodine scanning in the first stages of the disease. In patients who present with pulmonary metastasis, involvement of the cervical lymph node and distant metastasis to other regions are more likely to occur.

To date, few, if any studies, have investigated the disease course in DTC patients with pulmonary metastasis, and these studies have mainly focused on the response to treatment and survival. In this study, we comprehensively assessed the disease course, including the number of radioiodine therapy sessions, the total cumulative dose that the patients received, and the amount of time required to respond to treatment. In previous studies, the 5- and 10-year survival rates were both mainly greater than $80 \%$, but for FTC patients, it was generally in the range of $40 \%$ to $50 \%$. In contrast, in our study, the 5- and 9-year survival rates were calculated as $76 \%$ and $51 \%$, respectively. The main reason for the discrepancy in the survival rates reported by different studies is that the proportion of FTC and PTC patients included in each study is different.

In our study, of the 75 patients who were followed up for an average of 40 months (range, 8-118 months), 14 (18.7\%) responded to treatment at the end of follow-up. Even though the proportion of patients with FTC and those with PTC differed between previous studies, in all the studies, the response to treatment was much poorer in FTC patients than in PTC patients. In the current study, there was no statistically significant difference in response to treatment between men and women and between patients with different patterns of pulmonary involvement; however, a significant difference in treatment response was found between PTC and FTC patients.

Several studies have been conducted to investigate the outcome and prognosis in DTC patients who present with pulmonary metastasis. For example, in a previous survey, a follow-up study was performed on 272 DTC patients over a 23 -year period. ${ }^{14}$ Of 70 patients with pulmonary metastasis, 30 died during follow-up, and only 4 achieved complete remission; furthermore, the 5-, 10-, $15-$, and 20-year survival rates were reported as $68.5 \%, 54 \%$, $41.6 \%$, and $27.7 \%$, respectively. In this previous survey, the most important prognostic factors were age, Tg levels after surgery, cumulative dose of radioiodine, and difference in the size of the tumor between groups with and without pulmonary metastases. Based on their findings, they concluded that pulmonary metastasis of FTC weakens prognosis. A retrospective study was performed over a period of more than 40 years to estimate the survival of 96 DTC patients with pulmonary metastases. It was observed that age younger than 45 years, absorption of radioiodine by metastatic tissue, and miliaric metastases were associated with longer survival. ${ }^{15}$ In another study, 17 patients with diffuse and focal pulmonary metastasis were followed up for 89 months to study their clinical presentation and response to treatment. ${ }^{16}$ After the follow-up, 16 patients survived, and only 1 patient died of unrelated causes. Furthermore, 6 patients completely recovered at the end of the follow-up, and the pulmonary metastasis disappeared completely in 8 patients. The required dose for the treatment of pulmonary metastasis with a diffuse pattern was more than that required for pulmonary metastasis with a focal pattern. The authors concluded that the prognosis of patients with PTC with small pulmonary metastases was good and that the Tg level and small tumor size were associated with a better prognosis. The results of another study performed on 106 FTC patients with metastasis showed that the 5-, 10-, and 15-year survival rates were $82 \%, 63.8 \%$, and $23.9 \%$, respectively; furthermore, tumor size and age of the patients at presentation were identified as the most important prognostic factors. ${ }^{17}$

Regarding the cause of death, the current study revealed respiratory failure as consequence of multiple pulmonary metastases to be the most common cause of death in the patients, which is matched with previous studies generally. In addition, there were

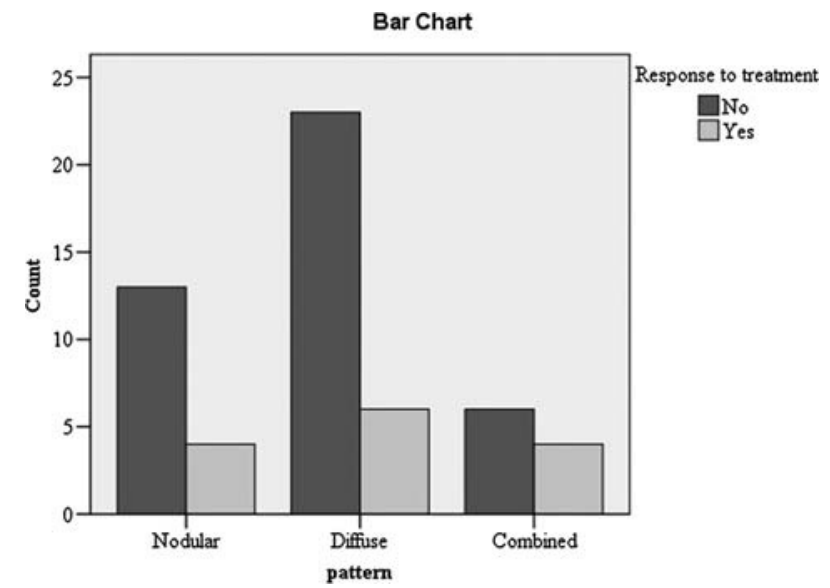

FIGURE 5. Response to radioiodine therapy in DTC patients according to the metastasis pattern. 
no significant differences in cause of death and other clinical and paraclinical parameters, which in our study could be due to the small number of patients who died. In a large study done by Kitamura et al, ${ }^{18}$ the causes of death in 161 fatal cases of thyroid cancer including 62 anaplastic carcinomas and 99 fatal differentiated carcinomas were analyzed. A single fatal condition was not delineated in 55 patients. In the remaining 106 cases, respiratory insufficiency (43\%) was the most common specific fatal cause, followed by circulatory failure (15\%), hemorrhage (15\%), and airway obstruction $(13 \%){ }^{18}$

At last, it should be highlighted that our study has some drawbacks. One of the limitations of the current study was the lack of pathologic data in the records of the patients. Another limitation was that the study duration was not sufficient for following up some of the patients to monitor the disease course and response to treatment. As DTC generally has a good prognosis, long follow-up periods are required for a better and comprehensive assessment of some of the consequences, such as death.

\section{CONCLUSIONS}

This study demonstrated that DTC patients with pulmonary metastasis have a relatively favorable prognosis and response rate, as well as long survival. It also indicated that the type of DTC is the only factor that affects the response to treatment in the studied group of DTC subjects.

\section{ACKNOWLEDGMENTS}

The authors thank their colleagues at their institutes for assistance with data acquisition.

\section{REFERENCES}

1. Schlumberger MJ. Papillary and follicular thyroid carcinoma. $N$ Engl J Med. 1998;338:297-306.

2. Davies L, Welch HG. Increasing incidence of thyroid cancer in the United States, 1973-2002. JAMA. 2006;295:2164-2167.

3. Olaleye O, Ekrikpo U, Moorthy R, et al. Increasing incidence of differentiated thyroid cancer in South East England: 1987-2006. Eur Arch Otorhinolaryngol. 2011;268:899-906.
4. Reynolds RM, Weir J, Stockton DL, et al. Changing trends in incidence and mortality of thyroid cancer in Scotland. Clin Endocrinol (Oxf). 2005;62: 156-162.

5. Hayashi Y, Lagarde F, Tsuda N, et al. Papillary microcarcinoma of the thyroid among atomic bomb survivors: tumor characteristics and radiation risk. Cancer. 2010;116:1646-1655.

6. Hayashi Y, Lagarde F, Tsuda N, et al. Radiation exposure does not significantly contribute to the risk of recurrence of Chernobyl thyroid cancer. J Clin Endocrinol Metab. 2010;96:385-393.

7. Jonklaas J, Sarlis NJ, Litofsky D, et al. Outcomes of patients with differentiated thyroid carcinoma following initial therapy. Thyroid. 2006;16:1229-1242.

8. Jonklaas J, Sarlis NJ, Litofsky D, et al. Pulmonary metastases in differentiated thyroid carcinoma. Study of 58 cases with implications for the primary tumor treatment. Cancer. 1984;53:982-992.

9. Brown AP, Greening WP, McCready VR, et al. Radioiodine treatment of metastatic thyroid carcinoma: the Royal Marsden Hospital experience. $\mathrm{Br} J$ Radiol. 1984;57:323-327.

10. Samaan NA, Schultz PN, Haynie TP, et al. Pulmonary metastasis of differentiated thyroid carcinoma: treatment results in 101 patients. J Clin Endocrinol Metab. 1985;60:376-380.

11. Elisei R, Molinaro E, Agate L, et al. Are the clinical and pathological features of differentiated thyroid carcinoma really changed over the last 35 years? Study on 4187 patients from a single Italian institution to answer this question. J Clin Endocrinol Metab. 2010;95:1516-1527.

12. Cho SW, Choi HS, Yeom GJ, et al. Long-term prognosis of differentiated thyroid cancer with lung metastasis in Korea and its prognostic factors. Thyroid. $2014 ; 24: 277-286$

13. Pitoia F, Bueno F, Cross G. Long-term survival and low effective cumulative radioiodine doses to achieve remission in patients with ${ }^{131}$ iodine-avid lung metastasis from differentiated thyroid cancer. Clin Nucl Med. 2014;39: 784-790.

14. Lin JD, Chao TC, Hsueh C. Follicular thyroid carcinomas with lung metastases: a 23-year retrospective study. Endocr J. 2004;51:219-225.

15. Ronga G, Filesi M, Montesano T, et al. Lung metastases from differentiated thyroid carcinoma. A 40 years' experience. $Q J$ Nucl Med Mol Imaging. 2004:48:12-19.

16. Kuo SF, Chen ST, Kao PF, et al. Papillary thyroid cancer with chest metastases only detected using radioactive iodine. Chang Gung Med J. 2004;27: 663-672.

17. Sugino K, Kameyama K, Nagahama M, et al. Follicular thyroid carcinoma with distant metastasis: outcome and prognostic factor. Endocr J. 2014;61: 273-279.

18. Kitamura Y, Shimizu K, Nagahama M, et al. Immediate causes of death in thyroid carcinoma: clinicopathological analysis of 161 fatal cases. J Clin Endocrinol Metab. 1999;84:4043-4049. 